\title{
Star Formation and the Metallicity Aversion of Long-Duration Gamma-Ray Bursts
}

\author{
John F. Graham ${ }^{1,2}$ and Andrew S. Fruchter ${ }^{1}$ \\ ${ }^{1}$ Space Telescope Science Institute, \\ 3700 San Martin Dr, Baltimore MD 21218, USA \\ ${ }^{2}$ Dept. of Physics and Astronomy, Johns Hopkins University, \\ 3400 N. Charles St, Baltimore MD 21218, USA
}

\begin{abstract}
It has been suggested that the apparent bias of long-duration GRBs (LGRBs) to low metallicity environments might be a result of the fact that star-formation is anti-correlated with metallicity. However, if this were the cause, one would expect other indicators of star formation, such as Type II and Type Ic SNe to demonstrate a similar bias. Here we show that local Type Ic and Type II SNe track the star-formation weighted metallicity distribution of the SDSS galaxies. In contrast LGRBs are typically found at far-lower metallicities than would be expected based on the distribution of star-formation. This is true even when one takes into account so-called "dark bursts". Indeed, while we will present data that show that some LGRBs form at very high metallicities, these objects enter the sample because of the large effective search volume produced by their bright hosts. The bias of LGRBs to low metallicity is real and must be related to a mechanism which is crucial in their formation.
\end{abstract}

Keywords. Gamma Rays: Bursts, Galaxy: Abundances, Stars: Formation.

While the existence of an apparent bias of long-duration GRBs (LGRBs) occurring in low metallicity environments is now generally accepted, Mannucci et al. (2011) proposes that this may be the byproduct of their "fundamental relation" that star-formation is anti-correlated with metallicity and not an intrinsic environmental preference in LGRB formation. Here we analyze across, LGRB \& SNe hosts as well as the general star forming galaxy population to determine whether low metallicity environments is indeed the intrinsic selection preference for LGRB formation. This work grew out of ongoing work looking at high metallicity LGRB host galaxies with a larger collaboration including Emily Levesque, Lisa Kewley, Jarle Brinchmann, Andrew Levan, Nial Tanvir, Sandy Patel, Greg Aldering, and Saul Perlmutter.

Beginning with updated populations in Modjaz et al. (2008) we make the following additions in populations and methodologies: Considering all LGRBs regardless of an observed associated SN event to increase the LGRB population to 14 bursts with 3 bursts being at high metallicity. Constraining the SDSS general star-forming galaxies and supernovae to a redshift of less then 0.04 to effectively transform our population from a magnitude to a volume limited sample. Introducing Type II SNe within this redshift range and selected from galaxies in the SDSS to provide a more numerous and consistently measured population of supernovae than the broad-lined type Ic population (see figure 2). Adopting a star-formation rate weighted sample of the general star-forming galaxies to graphically show the distribution of star-formation within our volume limited SDSS sample (again see figure 2). The latter was obtained by collecting all star-formation, selecting with a random number generator a specific bit of that star-formation and then selecting the specific galaxy that contributed it and repeating this process many times to collect a sample of galaxies based on their star-formation. Where previously it appeared that supernovae were biased toward bright host galaxies comparison, it is now visually 
apparent that both supernovae populations have the same star-formation distribution as the SDSS.

To provide a more analytical comparison we devise a method for directly comparing the populations on a normalized cumulative distribution plot (figure 4). For the star-forming SDSS galaxies within the redshift range mandated in the preceding paragraph we use their normalized cumulative star-formation. Assuming that LGRB and supernovae track the star-formation in which they occur this can be directly compared to the sorted fraction of LGRB and supernovae hosts vs. their increasing metallicities. (Note: this does not require any estimates of the SFR for the LGRB or supernovae hosts). For both non LGRB broad-lined type Ic supernovae and Type II SNe within this redshift range of the SDSS these host populations track the distribution of star-forming SDSS galaxies quite well suggesting that there is no additional metallicity constraint on the supernovae formation. The LGRBs however display a profound preference for lower metallicities than the other populations. Excluding the three high metallicity LGRB hosts the remainder of the population occur at metallicities containing only 5 to $10 \%$ of the star-formation. The exceedingly good match of the supernovae populations to the available star-formation excludes argument that the formation of LGRB progenitors is anti-correlated with metallicity. Thus the metallicity disparity between LGRB and broad-lined type Ic supernovae, as originally shown in Modjaz et al. (2008), is critical to the LGRB formation process.

While the preceding methodology looks at the distribution in metallicity, the starformation of the LGRB or supernovae hosts is assumed to be typical. To check this assumption we proceed to directly compare the distribution in star-formation rates of the LGRB and Type II SNe hosts to the general star-forming SDSS galaxy population. This comparison is performed by taking the star-formation rate of each LGRB and SN host and asking what fraction of the total star-formation in the general SDSS galaxy population occurs in galaxies with less star-formation than the target to generate a fractional value of total star-formation for each LGRB and SN host. These fractional values are then sorted and plotted against the fraction of each object type. Should this distribution of star-formation within each object type follow the general star-forming SDSS galaxy population then an LGRB host with its star-formation at, e.g., the top of the bottom quartile would be expected to be about consistent with $25 \%$ of the starformation in the general SDSS galaxy population and so on, such that the population tracks a diagonal line. Naturally this requires that for the Type II supernovae where the redshift is constrained to be low the general SDSS galaxy population is also so constrained and conversely for the LGRBs where no redshift constraint is added that the general SDSS galaxy population span the full range of the SDSS (the actual difference in the redshift ranges, while a source of error, is small since the bias towards the starformation being grossly dominated by the brightest objects is well established in both redshift ranges). As seen in figure 5, both the LGRBs and our larger general Type II SNe population track the diagonal well, indicating a good correspondence between the starformation rates of the two host populations and the general SDSS galaxy population, and suggesting that star-formation rate is correlated with LGRB and SNe formation. Curiously an attempt to generate a Type II SNe population from untargeted surveys failed this test quite badly and we believe it to be an issue with the Supernovae Factory to detect $\mathrm{SNe}$ in high surface brightness backgrounds.

From these analyzes we conclude that the low host metallicities observed for LGRBs are the direct consequence of an intrinsic preference for low metal content in the progenitor system during typical LGRB formation. This is consistent with the myriad of theoretical models requiring high progenitor rotation to which metal accelerated mass loss would likely be detrimental. The small subset of LGRBs found to occur in high metallicity hosts 
Fig. 1 to 3.- Metallicity vs. B band absolute galaxy luminosity of LGRB (squares) and broadlined Type Ic SNe hosts (circles). In the background SDSS (small points forming a cloud) and TKRS (small diamonds) galaxies are shown. SNe found through targeted galaxy surveys are not filled.

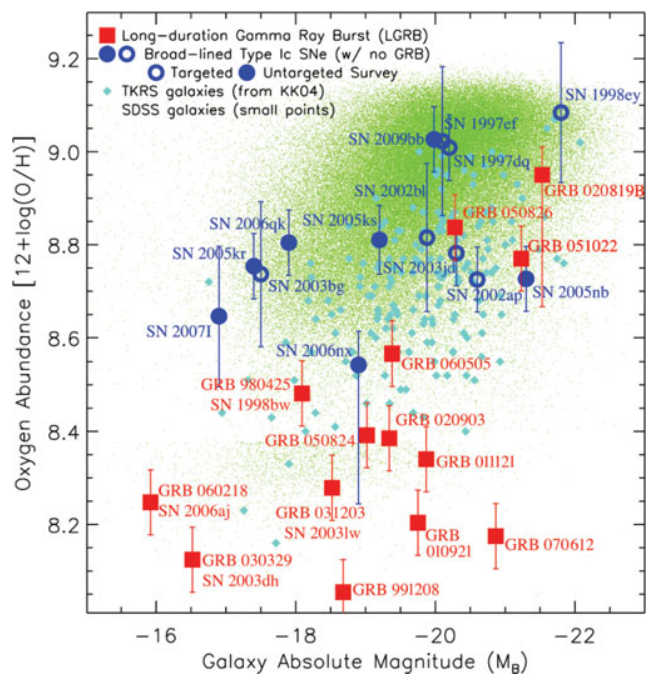

Figure 1. Site metallicities of LGRBs \& SNe. While using site metallicities, initially done in Modjaz et al. (2008), is ideal for comparing the progenitor metallicities of LGRB and SNe events it introduces a bias when comparing with general galaxy populations such as the SDSS \& TKRS populations thus we switch to using central metallicity in subsequent figures and analysis.

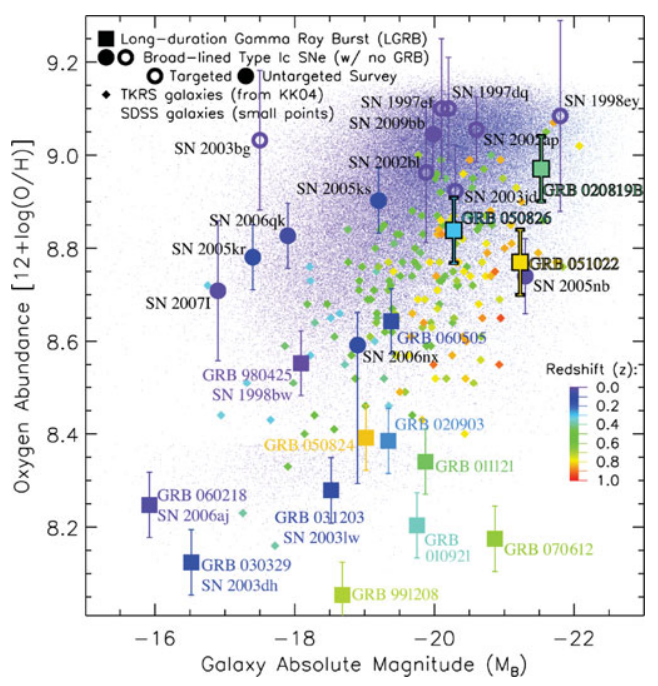

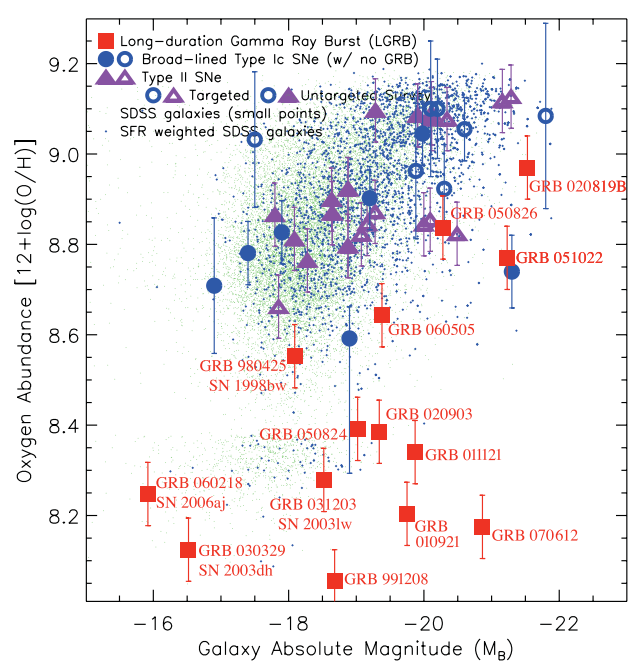

Figure 2. Central metallicities in a 0.0209 $<\mathrm{z}<0.04$ redshift cut to convert the SDSS into a volume limited sample. Since cutting the broad-lined Ic's would unacceptably degrade the sample size and they are already at low redshift we retain them and introduce a type II SNe population (purple triangles) strictly within the redshift range. LGRBs are shown purely for reference. We also select a subset of the redshift cut SDSS population in a star-formation weighted random manner to provide a graphical representation of the star-formation distribution of the SDSS. Note that this matches both SNe populations quite well as plotted in figure 4 .

Figure 3. Central metallicity of all objects with color now showing redshift. This provides a graphical representation of the effect of redshift on the luminosity metallicity relation. Note that the three high metallicity LGRBs (highlighted) are at typical metallicity for galaxies of their luminosity and redshift. This is not consistent with the otherwise observed LGRB metal aversion. Note: The color version of this figure (where color indicates redshift) is available only in the online proceedings.

and presumably with similarly high metallicity progenitors objects enter the sample due to the large effective search volume produced by their bright hosts and the order of magnitude larger amount of star-formation available at these higher metallicities. Since the non LGRB broad-lined type Ic supernovae population is consistent with the comparably low redshift subset of the general star-forming galaxy population, the LGRBs consistency 
with the general star-forming galaxy population by proxy excludes IMF differences or, as argued by Mannucci et al. (2011), star-formation being anti-correlated with metallicity as an explanation of the LGRB populations low metallicity. Thus the bias of LGRBs to low metallicity is real and must be related to a mechanism which is crucial in their formation.

\section{References}

Mannucci, F., Salvaterra, R., \& Campisi, M. A. 2011, MNRAS, 414, 1263

Modjaz, M., Kewley, L., Kirshner, R. P., et al. 2008, AJ, 135, 1136

Prieto, J. L., Stanek, K. Z., \& Beacom, J. F. 2008, ApJ, 673, 999

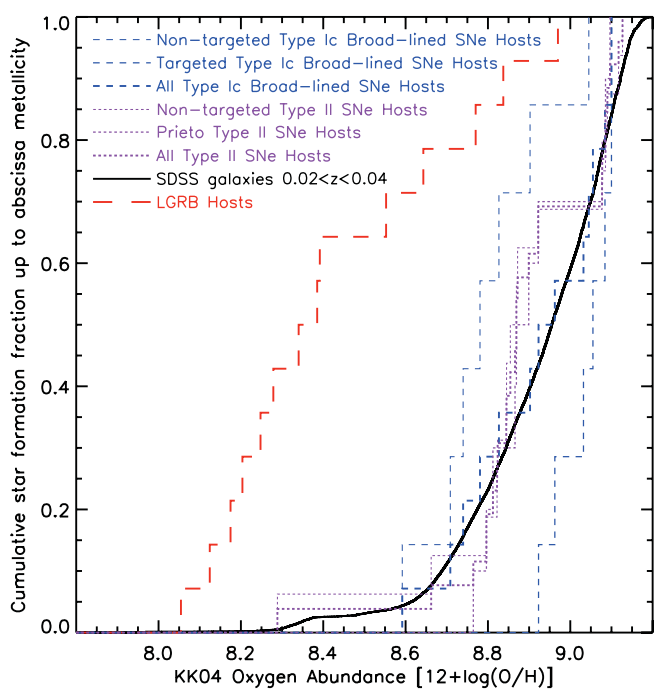

Figure 4. Star-formation cumulative summation fraction plots vs. core metallicity for the redshift range of 0.0209 to 0.04 with LGRB and SNe populations overplotted. LGRB host galaxies are shown in red, broad-lined Type Ic SNe hosts in blue, and Type II SNe hosts in purple. For the later two populations, the non targeted population is shown with a dashed line, the targeted (or potentially targeted population with a dotted line and the union of both with a solid line. The SDSS star-formation galaxy population is shown in back. Only the SDSS galaxies, including both the general star-forming galaxy and the type II SNe host populations are limited to the redshift cut range. No such cuts are applied to the LGRB or broad-lined type Ic $\mathrm{SNe}$ populations. While omitted for clarity using the site metallicity values for the broad-lined Type Ic supernovae populations does not shift any of those consistent with the LGRBs. This strongly suggests that LGRB hosts prefer host galaxies of metallicity considerably lower than would be obtained simply by following star-formation while both types of supernova are consistent with the star-formation distribution.

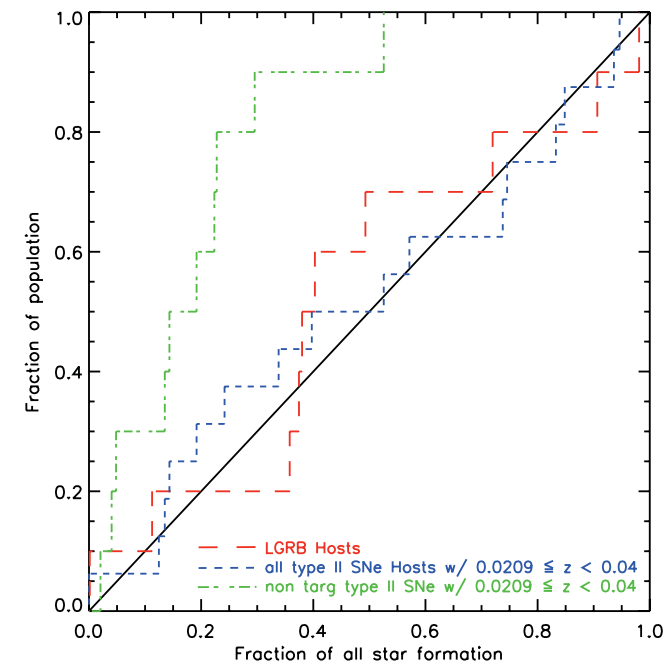

Figure 5. Normalized cumulative distribution plot of star-formation fraction values for various object classes. The abscissa values are obtained by determining for each LGRB and SN the fraction of total star-formation occurring in galaxies with lower star-formation rates than the objects host galaxy. For the type II SNe the star-formation of a volume limited SDSS sample was used spanning the same redshift range as the SNe sample whereas for the LGRBs the entire SDSS sample was used to match the selection biases in the LGRB host observations. The Prieto et al. (2008) type II SNe sample (blue line - "All" in the legend refers to this sample including SNe found through both targeted and non-targeted surveys and does not include the other dedicated non-targeted sample) tracks the diagonal reference line with astonishingly good accuracy. The LGRB sample (red line) also tracks the diagonal quite well indicating that the LGRB host galaxy sample has a typical star-formation distribution. Thus only metallicity itself remains to explain the discordant results shown in figure 4. Unfortunately however a similar comparison of the non targeted supernovae sample (green line) shows a clear off diagonal disagreement due to what we suspect is a failure of the Supernovae Factory to detect $\mathrm{SNe}$ in high surface brightness backgrounds. 


\section{Discussion}

PERLEY: I think it's now becoming fairly convincing that GRBs can occur even at high metallicity, but also that they do so at a much lower rate than at lower metallicity. So the next step is to try to quantify this trend. Do you have an estimate of what the high metallicity ( say $>0.5 \mathrm{Z}_{\odot}$ ) rate might be relative to the lower metallicity rate?

Graham: Correct on the lower rate \& next step. Also such rate estimate should be relative to the available star formation in the metallicity range. The small number of high metallicity LGRBs makes me unwilling to give a solid number but it looks like high metallicity LGRB formation is suppressed by at least a factor of five and probably more than one and less than two orders of magnitude compared with low metallicity LGRB formation (relative to the underlying available star formation at those metallicities).

FYNBO: Is the completeness of the SDSS uniform across the luminosity range you use and if not does this affect your analysis.

GRAHAM: The $0.02>\mathrm{z} \geqslant 0.04$ redshift cut SDSS sample provides reasonable luminosity completeness for galaxies brighter than $\mathrm{M}_{B}-18$ mag. The fraction of LGRBs, SNe \& SDSS star formation in fainter galaxies is roughly consistent and small - thus unlikely to be significantly biasing. 